\title{
Occurrence of Pasteurella multocida among pigs with respiratory disease in China between 2011 and 2015
}

\author{
Huisheng Liu', Zhanqin Zhao ${ }^{1 *}$, Xiaojian Xi', Qiao Xue ${ }^{1}$, Ta Long $^{1}$ and Yun Xue ${ }^{2}$
}

\begin{abstract}
Background: Prior to the 1990s, P. multocida capsular serogroup A was the most prevalent in China, followed by serogroups B and D. Thirty years later, serogroup D became the most prevalent, followed by serogroups A and B. However, the $P$. multocida capsular serogroups currently circulating in China remain unclear. Therefore, the aim of the present study was to provide an update on P. multocida serogroups isolated from diagnostic samples collected from clinically diseased pigs in Central and Eastern China from 2011 to 2015.

Results: Between February 2011 and October 2015, 296 isolates of Pasteurella multocida were collected from 3212 pigs with clinical respiratory disease in 12 provinces of China (isolation rate of 9.2\%). Of the 296 collected isolates, 146 (49.3\%) were P. multocida capsular type A, 141 (47.6\%) were capsular type D, and one was capsular type B. Streptococcus suis (94/193; 48.7\%), Haemophilus parasuis (76/193; 39.3\%), Escherichia coli (53/193; 27.5\%), and Bordetella bronchiseptica (26/193; 13.5\%) were frequently isolated together with P. multocida. A total of 14 toxigenic $P$. multocida strains co-isolated with other pathogens from 32 cases of atrophic rhinitis were classified into serogroup D. The virulence of P. multocida capsular type A isolates was higher than that of capsular type D isolates based on $\mathrm{LD}_{50}$ studies in mice.
\end{abstract}

Conclusions: Over the past 5 years, P. multocida capsular type A was the most frequently isolated from diagnostic submissions in Central and Eastern China, followed by serogroups D and B.

Keywords: Pasteurella multocida, Bordetella bronchiseptica, Swine, Respiratory pathogen, Co-infection

\section{Background}

Pasteurella multocida (P. multocida), a Gram-negative, non-motile, facultative coccobacillus, belonging to the Pasteurellaceae family [1], is an opportunistic pathogen and common inhabitant of the upper respiratory tract of many animal species, and a causative agent of numerous economically important diseases worldwide, including atrophic rhinitis (AR) in swine, fowl cholera in birds, pneumonia and shipping fever in cattle, snuffles in rabbits [2-4], and occasional zoonotic infections in humans [5].

P. multocida strains are classified into five capsular serogroups (A, B, D, E, and F) and 16 somatic serotypes (1 to 16) based on lipopolysaccharide antigens [6-8]. To date, capsular serogroups A, B, and D have been identified

\footnotetext{
* Correspondence: zhaozhanqin@126.com

'Laboratory of Veterinary Microbiology, College of Animal Science and

Technology, Henan University of Science and Technology, Luoyang, China

Full list of author information is available at the end of the article
}

in pigs $[8,9]$. Of these, serogroups $\mathrm{A}$ and $\mathrm{D}$ are causative agents of AR, which result in significant economic losses to the swine industry worldwide [9-12].

P. multocida possesses various virulence factors, including fimbriae, adhesins, and toxins, especially dermonecrotic toxin. Of these virulence factors, toxins produced by serogroups A and D play important roles in the pathogenicity of $P$. multocida. Only toxigenic strains can cause AR, which is characterized by loss of the nasal turbinate bone $[13,14]$. Therefore, data on P. multocida toxins can provide better understanding of pasteurellosis in pigs.

Diseases caused by different serogroups and their prevalence may change over time within particular regions. Prior to the 1990s, capsular serogroups A and B were the most prevalent in diseased pigs in China, followed by serogroup $\mathrm{D}$ [15]. Thirty years later, serogroups A and D became the most prevalent, followed by serogroup B [8]. However, 
these data are from several years ago and do not represent $P$. multocida capsular serogroups circulating in China in recent years, as the identification of $P$. multocida capsular serogroups currently circulating in China remain unclear. Hence, to provide an update on P. multocida capsular serogroups currently circulating in China, we investigated a total of $296 \mathrm{P}$. multocida strains isolated from pigs submitted to our diagnostic laboratory from 12 provinces of China from 2011 to 2015 to estimate the occurrence of $P$. multocida alone and in combination with other bacterial pathogens in pigs with respiratory disease. The findings of this study provide useful data to inform control strategies for respiratory disease and are relevant to future work on the development of effective vaccines.

\section{Methods}

\section{Samples collection}

Between February 2011 and October 2015, a total of 3212 lung samples were collected from pigs submitted to our diagnostic laboratory from 12 different provinces in Central and Eastern China for further identification of P. multocida. The data obtained from clinically affected pigs in Central and Eastern China may reflect, at least in part, the true occurrence of $P$. multocida in diagnostic submissions from the whole of China, since most of the pigs were raised in Central and Eastern China. All lung samples exhibited different degrees of pneumonic lesions and were collected by a single skilled veterinarian.

A total of 713 commercial pig farms were included, which had a wide variety of management types and herd size with small-, medium- and large-scale pig farms as well as various types of backyard farms. Only one pig farm was selected per region. If there was more than one pig farm in the same region, the larger pig farm was selected. During the study period, 13 to 107 pig cases were collected per month. One to 10 lung samples were randomly collected per herd. If more than one $P$. multocida isolate was found in the same herd, one of the isolates belonging the same serogroup was included in the analysis. All samples were processed for bacterial culture within $10 \mathrm{~h}$ of collection.

\section{Bacterial isolation and identification}

Each sample was plated on tryptic soy agar supplemented with $10 \mu \mathrm{g} / \mathrm{mL}$ of nicotinamide adenine dinucleotide and $5 \%$ fetal calf serum. All plates were incubated at $37^{\circ} \mathrm{C}$ for 24-48 h. Afterward, the isolates were purified and cultured by standard methods for rapid primary identification of $P$. multocida, Streptococcus suis (S. suis), Haemophilus parasuis (HPS), Escherichia coli (E. coli), Bordetella bronchiseptica $(B b)$, and Salmonella spp., respectively, by polymerase chain reaction (PCR) using the respective specific primers listed in Table 1, as described previously
[16-21]. After this stage, the strains were further identified by Gram-staining characteristics and oxidase (Gramnegative bacilli) or catalase tests [22]. All primers were synthesized by Sangon Biotech Co., Ltd. (Shanghai, China). Standard P. multocida, E. coli, and Salmonella strains, purchased from China Institute of Veterinary Drug Control (Beijing, China), and $B b$ and HPS strains, stored at our laboratory [23], were used as positive controls. Sterile water was used as a negative control. All isolates were freeze-dried and stored at $-80{ }^{\circ} \mathrm{C}$.

\section{Capsule typing}

The capsular types of $P$. multocida were confirmed by multiplex capsule PCR with capsule-specific primer pairs (Table 1), as described by Townsend et al. [7].

\section{Detection of toxA gene}

The toxA gene of $P$. multocida was confirmed by PCR with a specific primer pair (Table 1), as described by Zhao et al. [24].

\section{Detection of virulence}

Twenty-five BALB/c mice at 6 weeks of age (Vital River Laboratories Co., Ltd., Beijing, China) were randomly assigned to five groups (A1-A5) of five animals each, as previously described [25]. The mice were housed in a standard animal facility with ad libitum access to normal rodent diet and water. P. multocida was cultured on trypticase soy broth supplemented with $5 \%$ fetal calf serum and incubated on a shaking table at $200 \mathrm{rpm}$ and temperature of $37{ }^{\circ} \mathrm{C}$ for $12-16 \mathrm{~h}$. The number of $P$. multocida were enumerated by plate counts. Cultures were serially diluted 5 -fold in sterile phosphate buffered saline (PBS). Then, the original cultures (undiluted), cultures diluted 5-fold, cultures diluted 25-fold, and cultures diluted 125-fold were generated. The mice in groups A1-A4 were inoculated intraperitoneally with $0.2 \mathrm{ml}$ per mouse of the original cultures, and cultures diluted 5,25 , and 125 -fold, respectively, and the mice in group A5 were injected with $0.2 \mathrm{ml}$ of sterile PBS, as a control group. The $50 \%$ lethal dose $\left(\mathrm{LD}_{50}\right)$ was calculated on 15 days post-infection, as previously described [25].

\section{Statistical analysis}

Statistical analysis was performed using SPSS version 17.0 software (SPSS Inc., Chicago, IL, USA). A $p$-value $<0.05$ was considered statistically significant.

\section{Results}

Prevalence of $P$. multocida in porcine clinical samples

Over the 5-year period of this study, P. multocida was isolated from 296/3212 (9.2\%) lung samples collected from pigs with clinical respiratory disease. The annual isolation rates ranged from $7.5 \%$ to $10.7 \%$ with the 
Table 1 Primers used for the identification of bacterial isolates, capsule typing, and the toxA gene

\begin{tabular}{|c|c|c|c|c|c|}
\hline Strains & Target genes & Name & Sequences $\left(5^{\prime} \rightarrow 3^{\prime}\right)$ & Product (bp) & References \\
\hline \multirow[t]{2}{*}{ P. multocida } & \multirow[t]{2}{*}{ kmt1 } & $\mathrm{Pm}-1$ & ATCCGCTATTTACCCAGTGG & \multirow[t]{2}{*}{457} & \multirow[t]{2}{*}{ [17] } \\
\hline & & $\mathrm{Pm}-2$ & GCTGTAAACGAACTCGCCAC & & \\
\hline \multirow[t]{2}{*}{ P. multocida (serotype A) } & \multirow[t]{2}{*}{ hyaD-hyaC } & APm-1 & GATGCCAAAATCGCAGTCAG & \multirow[t]{2}{*}{1048} & \multirow[t]{2}{*}{ [7] } \\
\hline & & APm-2 & TGTTGCCATCATTGTCAGTG & & \\
\hline \multirow[t]{2}{*}{ P. multocida (serotype B) } & \multirow[t]{2}{*}{$\mathrm{dcbD}$} & BPm-1 & CATTTATCCAAGCTCCACC & \multirow[t]{2}{*}{758} & \multirow[t]{2}{*}{ [7] } \\
\hline & & $\mathrm{BPm}-2$ & GCCCGAGAGTTTCAATCC & & \\
\hline \multirow[t]{2}{*}{ P. multocida (serotype D) } & \multirow[t]{2}{*}{ dcbF } & DPm-1 & TTACAAAAGAAAGACTAGGAGCCC & \multirow[t]{2}{*}{647} & \multirow[t]{2}{*}{ [7] } \\
\hline & & DPm-2 & CATCTACCCACTCAACCATATCAG & & \\
\hline \multirow[t]{2}{*}{ P. multocida (serotype E) } & \multirow[t]{2}{*}{ ecbJ } & EPm-1 & TCCGCAGAAAATTATTGACTC & \multirow[t]{2}{*}{512} & \multirow[t]{2}{*}{ [7] } \\
\hline & & EPm-2 & GCTTGCTGCTTGATTITGTC & & \\
\hline \multirow[t]{2}{*}{ P. multocida (serotype F) } & \multirow[t]{2}{*}{$f c b D$} & $\mathrm{FPm}-1$ & AATCGGAGAACGCAGAAATCAG & \multirow[t]{2}{*}{852} & \multirow[t]{2}{*}{ [7] } \\
\hline & & FPm-2 & TTCCGCCGTCAATTACTCTG & & \\
\hline \multirow[t]{2}{*}{ P. multocida (toxA gene) } & \multirow[t]{2}{*}{ toxA } & toxA-1 & CTTAGATGAGCGACAAGG & \multirow[t]{2}{*}{864} & \multirow[t]{2}{*}{ [24] } \\
\hline & & toxA-2 & GAATGCCACACCTCTATAG & & \\
\hline \multirow[t]{2}{*}{ S. suis } & \multirow[t]{2}{*}{ gdh } & Ss-1 & GCAGCGTATTCTGTCAAACG & \multirow[t]{2}{*}{689} & \multirow[t]{2}{*}[20]{} \\
\hline & & Ss-2 & CCATGGACAGATAAAGATGG & & \\
\hline \multirow[t]{3}{*}{ HPS } & $16 \mathrm{~S}$ rRNA & HPS-1 & TATCGRGAGATGAAAGAC & 1086/1090 & [21] \\
\hline & & HPS-1' & GTAATGTCTAAGGACTAG & & \\
\hline & & HPS-2 & CCTCGCGGCTTCGTC & & \\
\hline E. coli & uidA & Ec-1 & ATGAAAGCTGGCTACAGGAAGGCC & 264 & [16] \\
\hline & & Ec-2 & GGTTTATGCAGCAACGAGACGTCA & & \\
\hline$B b$ & flaB & $\mathrm{Bb}-1$ & TGGCGCCTGCCCTATC & 237 & [19] \\
\hline & & $\mathrm{Bb}-2$ & AGGCTCCCAAGAGAGAAA & & \\
\hline Salmonella & inva & Sal-1 & CAGGATACCTATAGTGCTGC & 580 & [18] \\
\hline & & Sal-2 & CGCACCGTCAAAGGAACCGT & & \\
\hline
\end{tabular}

S. suis, HPS, E. coli, and Bb represent Streptococcus suis, Haemophilus parasuis, Escherichia coli and Bordetella bronchiseptica, respectively

highest isolation rate recorded in 2012 and the lowest in 2011 (Table 2). Differences in the isolation rates were not significant $(p>0.05)$ between February 2011 and October 2015. There was no association between collection month and isolation rate $(p>0.05)$. The monthly isolation rates ranged from $7.4 \%$ to $11.8 \%$ with the highest recorded in month 12 , followed by months 6, 11, and 9 (Fig. 1). In addition, strains of $P$. multocida were isolated throughout the year without seasonal variation $(p>0.05$; spring, months $3-5$; summer, months 6-8; autumn, months 9-11; winter, months 12-2; Fig. 1).

Table 2 Numbers of isolates of $P$. multocida and other pathogens detected in 3212 lung samples from pigs collected between February 2011 and October 2015

\begin{tabular}{|c|c|c|c|c|c|c|c|c|c|}
\hline \multirow[t]{2}{*}{ Year } & \multirow{2}{*}{$\begin{array}{l}\text { No. of } \\
\text { sample }\end{array}$} & \multirow{2}{*}{$\begin{array}{l}\text { No. of } P \text {. } \\
\text { multocida }\end{array}$} & \multirow{2}{*}{$\begin{array}{l}\text { Isolation } \\
\text { rates(\%) }\end{array}$} & \multicolumn{6}{|c|}{ Pathogens co-infected with $P$. multocida } \\
\hline & & & & S.suis & HPS & E. coli & $B b$ & Salmonella & Others \\
\hline 2011 & 616 & 46 & 7.5 & 15 & 13 & 8 & 3 & 2 & 2 \\
\hline 2012 & 570 & 61 & 10.7 & 20 & 17 & 11 & 4 & 2 & 4 \\
\hline 2013 & 630 & 57 & 9.0 & 19 & 15 & 11 & 6 & 1 & 3 \\
\hline 2014 & 754 & 72 & 9.6 & 22 & 17 & 12 & 7 & 3 & 6 \\
\hline 2015 & 642 & 60 & 9.3 & 18 & 14 & 11 & 6 & 2 & 4 \\
\hline \multirow[t]{2}{*}{ Total } & 3212 & 296 & 9.2 & 94 & 76 & 53 & 26 & 10 & 19 \\
\hline & & & & (48.7\%) & (39.3\%) & $(27.5 \%)$ & $(13.5 \%)$ & (5.1\%) & (9.8\%) \\
\hline
\end{tabular}

aThere were no significant differences in the annual isolates rates of $P$. multocida between 2011 and 2015 ( $p>0.05)$. Statistical analysis was performed using the $X^{2}$ test. S. suis, HPS, E. coli, and Bb represent Streptococcus suis, Haemophilus parasuis, Escherichia coli and Bordetella bronchiseptica, respectively 


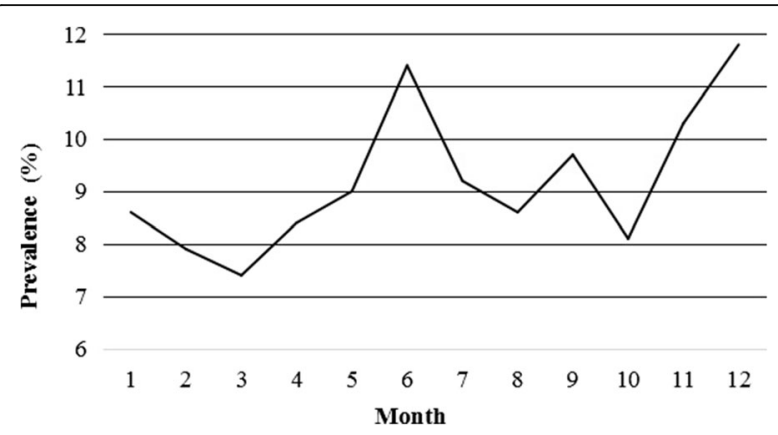

Fig. 1 Distribution of $P$. multocida isolates by the sampling month between February 2011 and October 2015. The isolation rates were 19/222, 20/253, 22/296, 25/297, 21/233, 38/332, 28/303, 24/280, 21/ $217,21 / 260,29 / 281$, and $28 / 238$ for months $1-12$, respectively. The isolation rates given are the average isolation rates per month over the 5-years study period. The highest isolation rates were recorded in month 12, followed by months 6, 11, and 9. However, as a whole, differences in the isolation rates were not significant $(p>0.05)$. Statistical analysis was performed using the $x^{2}$ test

P. multocida strains of capsular type A were isolated from 146 lung samples (49.3\%), capsular type D strains were isolated from 141 lung samples (47.6\%), capsular type B strains were isolated from one lung sample, and eight isolates were untypeable. Capsular types $\mathrm{E}$ and $\mathrm{F}$ were not detected in this study.

Prevalence of $P$. multocida accompanied by other species P. multocida was isolated together with other bacterial pathogens in $65.2 \%(193 / 296)$ of the lung samples (Table 2). S. suis (94; 48.7\%), HPS (76; 39.3\%), and E. coli (53; 27.5\%) were most frequently isolated together with $P$. multocida, followed by $B b(26 ; 13.5 \%)$ and Salmonella spp. (10; 5.1\%). S. suis was isolated from $25.3 \%(812 / 3212)$ of the lung samples, HPS was isolated from $16.6 \%$ (533/3212), E. coli was isolated from $12.9 \%$ (415/3212), $B b$ was isolated from $7.3 \%$ (236/3212), and Salmonella was isolated from 3.3\% (107/ 3212). P. multocida was the fourth most frequently isolated bacteria after S. suis, HPS and E. coli.

\section{Prevalence of toxigenic $P$. multocida accompanied by other species}

Of the 296 P. multocida isolates, only 14 toxigenic strains of P. multocida were identified by PCR and all were classified as capsular type D. Over the 5-year period of this study, 32/3212 (1\%) pigs with typical clinical signs of AR were identified. All toxigenic strains of $P$. multocida were isolated from pigs with clinical signs of AR. Ten of these toxigenic $P$. multocida strains were isolated with $B b$, seven with HPS, three with $S$. suis, and one with E. coli. In addition, $B b$ was isolated from pigs with no infection of toxigenic $P$. multocida strains.

\section{Virulence}

Twenty strains of P. multocida of each capsular types A and $\mathrm{D}$ were randomly selected, respectively, to estimate the $\mathrm{LD}_{50}$ in mice. Twenty-five mice were used for each of these 40 isolates (1000 mice in total). The results showed that the virulence of only one isolate of $P$. multocida capsular type A was relatively weak with a $\mathrm{LD}_{50}$ of $2.1 \times 10^{5}$, and the virulence of PM-30 of P. multocida capsular type D strain was lowest with a $\operatorname{LD}_{50}$ of $>4.9 \times 10^{7}$. As a whole, the virulence of $P$. multocida capsular type A was higher than that of capsular type D (Table 3 ).

\section{Discussion}

In China, as in other parts of the world, P. multocida is frequently associated with outbreaks of respiratory infections in pigs. In this study, the prevalence of $P$. multocida infection was $9.2 \%(296 / 3212)$, which was higher $(p>0.05$, $\chi^{2}$ test) than in previous reports $(8.0 \%, 233 / 2912)$ from China [8]. Additionally, the $\chi^{2}$ test results showed that the annual prevalence of $P$. multocida infection was relatively stable $(p>0.05)$ between 2011 and 2015, in accordance with the annual prevalence from 2003 to 2007, as reported by Tang et al. [8]. The results of this study showed that the prevalence of $P$. multocida infection did not significantly differ $(p>0.05)$ by season, in agreement with the report by Tang et al. [8]. In addition, there was no clear association of $P$. multocida infection with the sampling month, with the highest prevalence in month 12 , followed by month 6 . However, because only a few samples fron each month were assayed, the data in this study may not reflect the real differences between months. Hence, the difference in isolation rates between months should to be addressed in future studies.

Our findings suggest that strains of $P$. multocida are widely prevalent on pig farms, and there were no differences in the prevalence ( $p>0.05, \chi^{2}$ test) of P. multocida of capsular type A (49.3\%) and D (47.6\%), which was different from the results of previous report from China $(39.5 \%$ vs. $54.9 \%, p<0.05)$ [8] and different from the report by Rajkhowa et al. [26]. These results showed that the prevalence of $P$. multocida capsular type A strains exhibited a tendency to increase over the past 5 years in China. The reasons for this tendency were unclear and need to be addressed in future studies. The eight untypeable isolates, which were associated with pneumonic pasteurellosis, should be further investigated.

In this study, S. suis, HPS, E. coli, and $B b$ were frequently isolated together with $P$. multocida. Similar results were reported by Tang et al. [8] and Zhao et al. [22]. Accumulating evidence suggests that mixed infection of two or more bacteria may have been common over the last decade in China. Therefore, the practical significance of this finding is that future work on control of disease caused by $P$. multocida in pigs may require investigation of multivalent vaccines based 
Table 3 Virulence of $P$. multocida capsular type $A$ and $D$ strains in mice

\begin{tabular}{|c|c|c|c|c|c|c|c|}
\hline Strain & Capsular type & Origin & $\mathrm{LD}_{50}$ (CFU) & Strain & Capsular type & Origin & $\mathrm{LD}_{50}$ (CFU) \\
\hline$\overline{P M-1}$ & $A$ & Henan & $<84$ & PM-21 & $\mathrm{D}$ & Henan & $1.3 \times 10^{6}$ \\
\hline PM-2 & A & Shanxi & $<23$ & PM-22 & $D$ & Hubei & $3.4 \times 10^{6}$ \\
\hline PM-3 & A & Hubei & $4.1 \times 10^{3}$ & PM-23 & D & Henan & $1.9 \times 10^{5}$ \\
\hline PM-4 & A & Henan & $<16$ & PM-24 & D & Sichuan & $2.1 \times 10^{5}$ \\
\hline PM-5 & A & Shanxi & $<38$ & PM-25 & D & Hubei & $2.6 \times 10^{4}$ \\
\hline PM-6 & A & Anhui & $<167$ & PM-26 & D & Shanxi & $2.6 \times 10^{4}$ \\
\hline PM-7 & A & Henan & $<68$ & PM-27 & D & Henan & $5.7 \times 10^{5}$ \\
\hline PM-8 & A & Sichuan & $<145$ & PM-28 & D & Henan & $2.2 \times 10^{6}$ \\
\hline PM-9 & $A$ & Shanxi & $<17$ & PM-29 & $D$ & Hubei & $3.3 \times 10^{6}$ \\
\hline PM-10 & A & Anhui & 104 & PM-30 & D & Henan & $>4.9 \times 10^{7}$ \\
\hline PM-11 & A & Henan & $7.2 \times 10^{4}$ & PM-31 & D & Shandong & $3.3 \times 10^{6}$ \\
\hline PM-12 & A & Shanxi & $<69$ & PM-32 & D & Henan & $1.6 \times 10^{7}$ \\
\hline PM-13 & A & Henan & $6.8 \times 10^{4}$ & PM-33 & $D$ & Sichuan & $5.5 \times 10^{5}$ \\
\hline PM-14 & A & Shanxi & $<103$ & PM-34 & D & Hubei & $2.9 \times 10^{5}$ \\
\hline PM-15 & A & Henan & 123 & PM-35 & $D$ & Henan & $1.2 \times 10^{7}$ \\
\hline PM-16 & A & Hubei & $<134$ & PM-36 & D & Shanxi & $1.3 \times 10^{5}$ \\
\hline PM-17 & A & Shanxi & $<70$ & PM-37 & $D$ & Henan & $1.6 \times 10^{5}$ \\
\hline PM-18 & A & Henan & $<42$ & PM-38 & D & Shandong & $5.3 \times 10^{5}$ \\
\hline PM-19 & A & Henan & $2.0 \times 10^{4}$ & PM-39 & $D$ & Henan & $4.4 \times 10^{4}$ \\
\hline PM-20 & A & Hubei & $<93$ & PM-40 & $D$ & Jiangsu & $5.4 \times 10^{5}$ \\
\hline
\end{tabular}

PM represent Pasteurella multocida

on these bacteria rather than a monovalent vaccine. In addition, our results showed high isolation rates of S. suis, HPS, E. coli, and $B b$, in accordance with recent reports from China $[8,22]$ and some other countries [27-29].

AR is seldom reported in China, which is supported by our findings (32/3212) as well as the reports by Tang et al. [8] and Zhao et al. [22]. The findings of previous studies combined with those of the present study confirmed the presence of AR in Henan, Shandong, Fujian, Hainan, Anhui, and Hubei provinces to date. In this study, all 14 toxigenic $P$. multocida strains were type D, which was in accordance with the findings of previous reports $[8,9,22,30]$. In addition, 14 toxigenic $P$. multocida strains were isolated together with one, two, or three other pathogens and $B b$ was most frequently isolated with toxigenic $P$. multocida.

Previous reports have mainly focused on the detection of virulence genes of $P$. multocida isolates. This is the first large-scale study of the virulence of $P$. multocida isolates based on $\mathrm{LD}_{50}$ studies in mice. Our findings showed that the virulence of $P$. multocida capsular type A isolates was higher than that of capsular type D isolates. The high virulence and prevalence of isolates of $P$. multocida capsular type A is expected to increase economic losses to the swine industry in China.

\section{Conclusions}

Our results disclosed epidemiological information of $P$. multocida infection of pigs over the past 5 years in China. Data from a total of 296 P. multocida isolates revealed no significant difference in the prevalence of $P$. multocida capsular types $\mathrm{A}$ and $\mathrm{D}$ in diagnostic procine lung samples submitted from 12 provinces in Central and Eastern China, and S. suis, HPS, E. coli, and $B b$ are often isolated together with $P$. multocida. In addition, our results showed that the virulence of $P$. multocida capsular type A was greater than that of capsular type D. The findings of this study are expected to facilitate a better understanding of the current status of $P$. multocida infection among pigs in China.

\section{Abbreviations}

AR: Atrophic rhinitis; Bb: Bordetella bronchiseptica; E. coli: Escherichia coli: HPS: Haemophilus parasuis; LD 50: 50\% lethal dose; P. multocida: Pasteurella multocida; PBS: Phosphate buffered saline; PCR: Polymerase chain reaction; S. suis: Streptococcus suis

\section{Acknowledgments}

We thank Kunpeng Chen and Le Wang for their excellent technical assistance.

\section{Funding}

This study was supported by grants from National Natural Science Foundation of China (no. 31302106), the Open Funds of State Key Laboratory of Veterinary Etiological Biology (no. SKLVEB2013KFKT009) and the Research and Development Foundation of Henan University of Science and Technology (no. 2015ZDCXY04). 


\section{Availability of data and materials}

Not applicable.

\section{Authors' contributions}

HSL analyzed the data and wrote the manuscript. ZQZ devised study, participated in its design. QX and XJX initiated the study and collected samples. TL and YX performed PCR analysis. All authors read and approved the final manuscript.

\section{Competing interests}

None of the authors has any financial or personal relationships that could inappropriately influence or bias the content of the paper.

\section{Consent for publication}

Not applicable.

\section{Ethics approval and consent to participate}

All animal procedures carried out in this study were performed in compliance with the guidelines of the Animal Care and Use Committee of Henan University of Science and Technology (No. DK2015EA036).

\section{Author details}

${ }^{1}$ Laboratory of Veterinary Microbiology, College of Animal Science and Technology, Henan University of Science and Technology, Luoyang, China. 2Laboratory of Medical Engineering, College of Medical Technology and Engineering, Henan University of Science and Technology, Luoyang, China.

Received: 8 April 2016 Accepted: 22 December 2016

Published online: 10 January 2017

\section{References}

1. Carter GR. Serological classification of Pasteurella multocida. Vet Rec. 1987; 121:382-3.

2. Hatfaludi T, Al-Hasani K, Gong L, Boyce JD, Ford M, Wilkie IW, Quinsey N, Dunstone MA, Hoke DE, Adler B. Screening of 71 P. multocida proteins for protective efficacy in a fowl cholera infection model and characterization of the protective antigen PIpE. PLoS One. 2012;7:e39973.

3. Illambas J, Potter T, Sidhu P, Rycroft AN, Cheng Z, Lees P. Pharmacodynamics of florfenicol for calf pneumonia pathogens. Vet Rec. 2013;172:340.

4. Crawshaw WM, Caldow GL. Field study of pneumonia in vaccinated cattle associated with incorrect vaccination and Pasteurella multocida infection. Vet Rec. 2015;176:434.

5. Bardou M, Honnorat E, Dubourg G, Couderc C, Fournier PE, Seng P, Stein A. Meningitis caused by Pasteurella multocida in a dog owner without a dog bite: clonal lineage identification by MALDI-TOF mass spectrometry. BMC Res Notes. 2015:8:626.

6. Rhoades KR, Rimler RB. Capsular groups of Pasteurella multocida isolated from avian hosts. Avian Dis. 1987:31:895-8.

7. Townsend KM, Boyce JD, Chung JY, Frost AJ, Adler B. Genetic organization of Pasteurella multocida cap loci and development of a multiplex capsular PCR typing system. J Clin Microbiol. 2001;39:924-9.

8. Tang X, Zhao Z, Hu J, Wu B, Cai X, He Q, Chen H. Isolation, antimicrobial resistance, and virulence genes of Pasteurella multocida strains from swine in China. J Clin Microbiol. 2009;47:951-8.

9. Davies RL, MacCorquodale R, Baillie S, Caffrey B. Characterization and comparison of Pasteurella multocida strains associated with porcine pneumonia and atrophic rhinitis. J Med Microbiol. 2003;52:59-67.

10. Dziva F, Muhairwa AP, Bisgaard M, Christensen H. Diagnostic and typing options for investigating diseases associated with Pasteurella multocida. Vet Microbiol. 2008;128:1-22.

11. Pors SE, Hansen MS, Christensen H, Jensen HE, Petersen A, Bisgaard M. Genetic diversity and associated pathology of Pasteurella multocida isolated from porcine pneumonia. Vet Microbiol. 2011;150:354-61.

12. Strack J, Heni H, Gilsbach R, Hein L, Aktories K, Orth JH. Noncanonical $\mathrm{G}$-protein-dependent modulation of osteoclast differentiation and bone resorption mediated by Pasteurella multocida toxin. MBio. 2014;5:e02190.

13. Djordjevic SP, Eamens GJ, Ha H, Walker MJ, Chin JC. Demonstration that Australian Pasteurella multocida isolates from sporadic outbreaks of porcine pneumonia are non-toxigenic (toxA-) and display heterogeneous DNA restriction endonuclease profiles compared with toxigenic isolates from herds with progressive atrophic rhinitis. J Med Microbiol. 1998;47:679-88.
14. Katsuda K, Hoshinoo K, Ueno Y, Kohmoto M, Mikami O. Virulence genes and antimicrobial susceptibility in Pasteurella multocida isolates from calves. Vet Microbiol. 2013;167:737-41.

15. Guo DH, Zheng M, Pan SN. Serological identification of capsular antigen of pasteurella multocida in China. Acta Veterinaria et Zootechinca Sinica. 1979; 02:67-8.

16. Bej AK, McCarty SC, Atlas RM. Detection of coliform bacteria and Escherichia coli by multiplex polymerase chain reaction: comparison with defined substrate and plating methods for water quality monitoring. Appl Environ Microbiol. 1991;57:2429-32.

17. Nagai S, Someno S, Yagihashi T. Differention of toxigenic from nontoxigenic isolates of Pasteurella multocida by PCR. J Clin Microbiol. 1994;32:1004-10.

18. Chiu CH, Ou JT. Rapid identification of Salmonella serovars in feces by specific detection of virulence genes, invA and spvC, by an enrichment broth culturemultiplex PCR combination assay. J Clin Microbiol. 1996;34:2619-22.

19. Hozbor D, Fouque F, Guiso N. Detection of Bordetella bronchiseptica by the polymerase chain reaction. Res Microbiol. 1999;150:333-41.

20. Okwumabua O, O'Connor M, Shull E. A polymerase chain reaction (PCR) assay specific for Streptococcus suis based on the gene encoding the glutamate dehydrogenase. FEMS Microbiol Lett. 2003;218:79-84.

21. Angen O, Oliveira S, Ahrens P, Svensmark B, Leser TD. Development of an improved species specific PCR test for detection of Haemophilus parasuis. Vet Microbiol. 2007;119:266-76

22. Zhao Z, Wang C, Xue Y, Tang X, Wu B, Cheng X, He Q, Chen H. The occurrence of Bordetella bronchiseptica in pigs with clinical respiratory disease. Vet J. 2011:188:337-40.

23. Xu HJ, Xue Y, Zhao ZQ, Chen KP, Wang L, Deng W. Comparative study on biological characterization of the epidemic serotypes haemophilus parasuis in China. Chin J Vet Sci. 2014;34:729-35.

24. Zhao ZQ, Pei J, Xue Y, Tang XB, Wu B, He H, Zhou XL, Cheng XC, He QG, Chen HC. Isolation, Identification and Characterization for Bordetella bronchiseptica from Diseased Pigs. Scientia Agricultura Sinica. 2008;41:4209-17.

25. Reed $\amalg$, Muench $\mathrm{H}$. A simple method of estimating fifty percent endpoints. Am J Hyg. 1938;27:493-7.

26. Rajkhowa S, Shakuntala I, Pegu SR, Das RK, Das A. Detection of Pasteurella multocida isolates from local pigs of India by polymerase chain reaction and their antibiogram. Trop Anim Health Prod. 2012:44:1497-503.

27. Katsumi M, Kataoka Y, Takahashi T, Kikuchi N, Hiramune T. Bacterial isolation from slaughtered pigs associated with endocarditis, especially the isolation of Streptococcus suis. J Vet Med Sci. 1997;59:75-8.

28. Maclnnes Jl, Gottschalk M, Lone AG, Metcalf DS, Ojha S, Rosendal T, Watson SB, Friendship RM. Prevalence of Actinobacillus pleuropneumoniae, Actinobacillus suis, Haemophilus parasuis, Pasteurella multocida, and Streptococcus suis in representative Ontario swine herds. Can J Vet Res. 2008;72:242-8.

29. Moorkamp L, Nathues H, Spergser J, Tegeler R, Beilage EG. Detection of respiratory pathogens in porcine lung tissue and lavage fluid. Vet J. 2008; 175:273-5.

30. Lariviere S, Leblanc L, Mittal KR, Martineau GP. Characterization of Pasteurella multocida from nasal cavities of piglets from farms with or without atrophic rhinitis. J Clin Microbiol. 1992;30:1398-401.

\section{Submit your next manuscript to BioMed Central and we will help you at every step:}

- We accept pre-submission inquiries

- Our selector tool helps you to find the most relevant journal

- We provide round the clock customer support

- Convenient online submission

- Thorough peer review

- Inclusion in PubMed and all major indexing services

- Maximum visibility for your research

Submit your manuscript at www.biomedcentral.com/submit
) Biomed Central 